\title{
Membrane Viscosity Determined from Shear-Driven Flow in Giant Vesicles
}

\author{
Aurelia R. Honerkamp-Smith, Francis G. Woodhouse, Vasily Kantsler, and Raymond E. Goldstein \\ Department of Applied Mathematics and Theoretical Physics, Centre for Mathematical Sciences, University of Cambridge, \\ Wilberforce Road, Cambridge CB3 OWA, United Kingdom
}

(Received 14 January 2013; published 17 July 2013)

\begin{abstract}
The viscosity of lipid bilayer membranes plays an important role in determining the diffusion constant of embedded proteins and the dynamics of membrane deformations, yet it has historically proven very difficult to measure. Here we introduce a new method based on quantification of the large-scale circulation patterns induced inside vesicles adhered to a solid surface and subjected to simple shear flow in a microfluidic device. Particle image velocimetry based on spinning disk confocal imaging of tracer particles inside and outside of the vesicle and tracking of phase-separated membrane domains are used to reconstruct the full three-dimensional flow pattern induced by the shear. These measurements show excellent agreement with the predictions of a recent theoretical analysis, and allow direct determination of the membrane viscosity.
\end{abstract}

DOI: 10.1103/PhysRevLett.111.038103

PACS numbers: 87.16.dm, 47.55.N-, 83.85.Cg

Ever since the work of Saffman and Delbrück on the dynamics of inclusions in biological membranes [1] it has been recognized that lipid bilayers can be viewed as ultrathin fluid layers endowed with a surface viscosity. Along with that of the surrounding fluid, this viscosity plays an important role in determining the translational and rotational diffusion constants of inclusions within the membrane [2]. A body of theoretical work [3,4] suggests that nonequilibrium dynamics of vesicles in external flows [5] can also be sensitive to the value of this viscosity [6]. As the membrane viscosity $\eta_{m}$ can be expressed as $d \times \eta$, where $d$ is the membrane thickness and $\eta$ is the bilayer fluid viscosity, the nanometric scale of $d$ renders $\eta_{m}$ very small. Not surprisingly, it has proven difficult to measure $\eta_{m}$; ingenious techniques that have been developed include measurements of the motion of membrane-bound microspheres [7], diffusion constants of domains in multicomponent membranes [8,9], and observation of fluctuation dynamics in membranes near a critical point $[10,11]$. Further afield, monolayers admit additional experimental techniques, including methods based on surface rheology [12] and microrheology methods such as observing dynamics of submerged optically trapped [13] or membranebound [14] microspheres. Rheological experiments have the advantage of being able to detect non-Newtonian behavior [15].

Interest in membrane dynamics also extends to flows within vesicles, especially in plant science, as the plant vacuole is contained within the vacuolar membrane (or tonoplast), which can comprise some of the largest lipid

Published by the American Physical Society under the terms of the Creative Commons Attribution 3.0 License. Further distribution of this work must maintain attribution to the author(s) and the published article's title, journal citation, and DOI. vesicles known: in internodal cells of the aquatic plant Chara corallina, these can be cylinders $1 \mathrm{~mm}$ in diameter and up to $10 \mathrm{~cm}$ long [16]. This tonoplast is subject to continuous hydrodynamic shear through the action of cytoplasmic streaming, motion of the cytoplasm surrounding the vacuole [17]. Because of its potential role in transport [18] there is great interest in the three-dimensional characteristics of such shear-induced flows [19] and the role played by the intervening tonoplast [20].

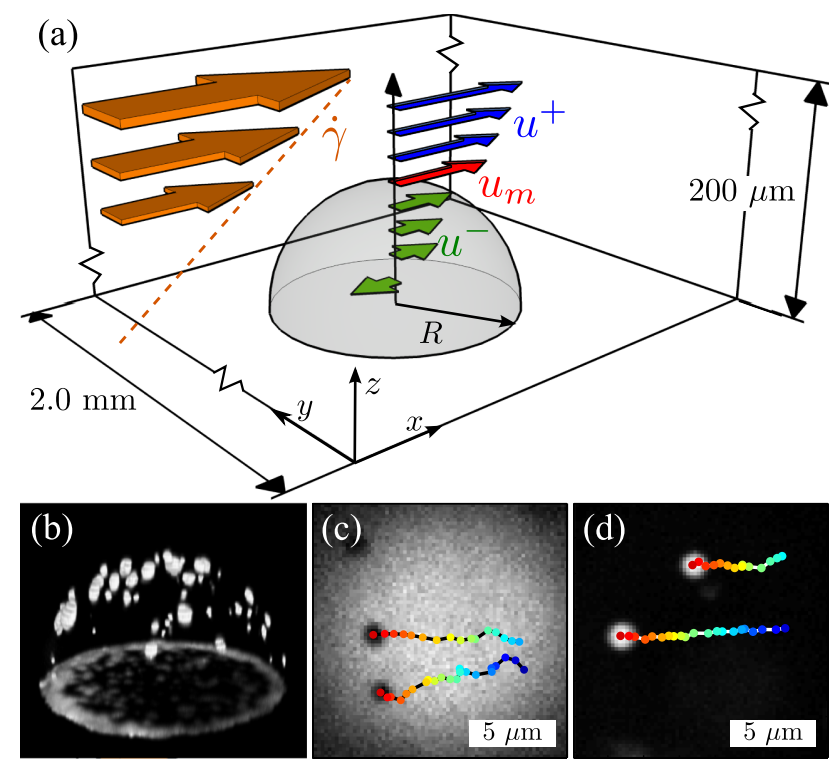

FIG. 1 (color online). Microfluidic shear experiment. (a) Schematic of the chamber (not to scale) and flows. (b) Confocal imaging reconstruction of an adhering hemispherical $L_{o}$ phase vesicle with small $L_{d}$ domains visible on its surface. (c)-(d) Tracking of gel domains in $L_{d}$ background (c) and $L_{d}$ domains in $L_{o}$ background (d), flowing across the vesicle apex at $\dot{\gamma}=2.6 \mathrm{~s}^{-1}$ (tracks color-coded in time over $\sim 2.6 \mathrm{~s}$ ). 
A key development in the study of membrane fluid dynamics was the conceptually simple experiment of Vézy et al. [21] (see also [22]) in which a vesicle was adhered to a solid surface and subjected to a simple shear flow. The flow induced in the membrane took the form of two vortices, rather than the simple overturning flow that would occur in a hemispherical droplet of one fluid in the background of an immiscible second fluid without the membrane $[23,24]$. This difference is attributable to the incompressibility of the membrane, which restricts the flow field to one that is two-dimensionally divergence free, i.e., area conserving, on the vesicle surface [25].

Since viscosity is the coefficient of proportionality between force per unit area on a surface and the adjacent shear rate, it is natural to ask whether the experimental setup of Vézy et al. [21] suggests a means to study membrane fluid mechanics in detail. To this end, we describe here a method that quantifies the flows set up by shear of adherent vesicles, and, through a recent calculation [25], provides a means of determining membrane viscosity. The method uses particle image velocimetry (PIV) to measure the three-dimensional flows inside and outside vesicles, and particle tracking to monitor the shear-induced movement of phase-separated domains within the membrane, in a microfluidic environment.

Figure 1 shows the experimental setup: a vesicle of radius $R$, typically in the range of $10-40 \mu \mathrm{m}$, adheres to the surface of a microfluidic chamber in the presence of a flow with shear rate $\dot{\gamma}$. The chamber, typically $2 \mathrm{~mm}$ wide and $200 \mu \mathrm{m}$ deep, is made from polydimethylsiloxane by soft lithography and sealed with a glass coverslip that had been treated to promote vesicle adhesion. Vesicles were produced by standard methods of electroformation [26] in $100 \mathrm{mM}$ sucrose with or without $0.5 \mu \mathrm{m}$ microspheres (Invitrogen, Carlsbad, CA). We chose lipid compositions to obtain two substantially different membrane viscosities. One composition gives primarily liquid-ordered $\left(L_{o}\right)$ vesicles with a small fraction of liquid-disordered $\left(L_{d}\right)$ phase at room temperature $\left(\sim 23^{\circ} \mathrm{C}\right)$ : $40 \mathrm{~mol} \%$ cholesterol (Sigma-Aldrich, St. Louis, MO), 55\% DPPC (dipalmitoylphosphatidylcholine), and 5\% DiPhyPC (diphytanoylphosphatidylcholine). DPPC, DOPC, and DiPhyPC were purchased from Avanti Polar Lipids (Alabaster, AL) and used without further purification. Vesicles containing primarily $L_{d}$ phase with a small fraction of gel domains were made from $85 \%$ DOPC and $15 \%$ DPPC. The $L_{d}$ phases were labeled with $0.5 \%$ TexasRedDPPE (Invitrogen). Coverslips were cleaned aggressively in $\mathrm{NaOH}$ and soaked in a solution of $0.001 \%$ polylysine for 30 minutes for use with $L_{d}$ phase vesicles, or in $0.0005 \%$ polyethylenimine for 5 minutes for use with $L_{o}$ phase vesicles. Vesicles were gently osmotically deflated by diluting into $130 \mathrm{mM}$ glucose and $10 \mathrm{mM}$ HEPES shortly before loading into the chamber.

Measurements were made on a Zeiss Cell Observer spinning disk confocal microscope with an electron-multiplied
CCD camera (Evolve, Photometrics, Tucson, AZ, $512 \times 512$ pixels), using an NA $1.4 / 63 \mathrm{X}$ oil-immersion objective. Flows were controlled by a syringe pump (PHD2000, Harvard Apparatus, Holliston, MA) and quantified by measuring far upstream from vesicles the speed of microspheres as a function of height above the coverslip. Shear rates were typically in the range $1 \leq \dot{\gamma} \leq$ $6 \mathrm{~s}^{-1}$. PIV was done with Matlab by adapting standard code [27] to track small dilute tracers by finding the time-averaged velocity field [28]. For three-dimensional reconstruction, movies were recorded at $\sim 30$ frames per second at intervals of $2-3 \mu \mathrm{m}$ throughout and above vesicles containing microspheres [Fig. 2(b)], giving two-dimensional velocity field slices [Fig. 2(a)]. From a stack of such slices a three-dimensional velocity field was
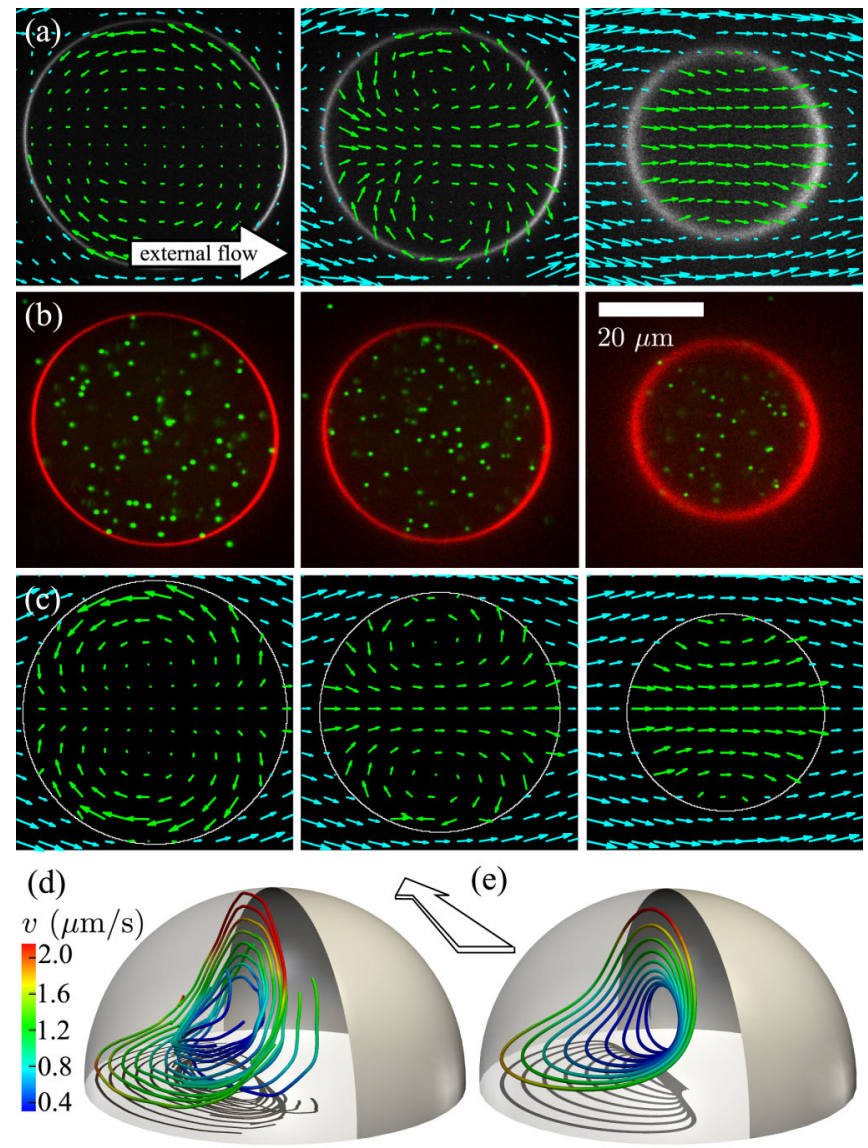

FIG. 2 (color online). Flow fields inside an adhering vesicle in shear. (a) Experimental two-dimensional PIV velocity fields at heights $z / R=0.26,0.47,0.71$ above coverslip. (b) Confocal slices at same fractional heights as (a) show vesicle (red) containing fluorescent microspheres. (c) Theoretical twodimensional velocity fields [25] for a sheared hemispherical vesicle at $z / R=0.3,0.5,0.7$. Interior and exterior PIV vectors in each panel of (a) and (c) have been rescaled for visual clarity. (d) Experimental streamlines of the three-dimensional velocity field obtained by integrating two-dimensional flow fields, compared with theory (e). Large arrows in (a), (d), and (e) indicate direction of imposed shear flow. 
determined from the incompressibility relation. Figure 2(d) shows a representative example of such streamlines.

To understand the flows set up in and around the vesicle, we distill the essential results of a recent calculation [25]. Assume that the vesicle is a hemispherical cap of radius $R$ and origin $x=y=z=0$, adhered to the plane $z=0$, and let $(r, \theta, \phi)$ be spherical polars centered at the origin. Given the fluid viscosity $\eta_{-}$inside the vesicle $(r<R)$, the membrane viscosity $\eta_{m}$, and the external fluid viscosity $\eta_{+}$ $(r>R)$, we wish to find three velocity fields: $\mathbf{u}^{-}$inside the vesicle, the two-dimensional flow $\mathbf{u}^{m}$ of the membrane, and $\mathbf{u}^{+}$outside the vesicle [Fig. 1(a)]. The two external flows obey the unforced Stokes and incompressibility equations, $\eta_{ \pm} \nabla^{2} \mathbf{u}^{ \pm}-\boldsymbol{\nabla} p^{ \pm}=\mathbf{0}$ and $\boldsymbol{\nabla} \cdot \mathbf{u}^{ \pm}=0$, with far-field asymptotics $\boldsymbol{u}^{+} \sim \dot{\gamma} z \hat{\boldsymbol{x}}$ as $r \rightarrow \infty$, the no-slip condition $\boldsymbol{u}^{ \pm}=\mathbf{0}$ on the plane $\theta=\pi / 2$, and no radial penetration, $\boldsymbol{u}^{ \pm} \cdot \hat{\boldsymbol{r}}=0$ at $r=R$. The three velocities must be continuous across the membrane, $\boldsymbol{u}^{+}=\boldsymbol{u}^{m}=\boldsymbol{u}^{-}$at $r=R$, and thus there is the planar no-slip condition $\boldsymbol{u}^{m}=\mathbf{0}$ at $\theta=\pi / 2$.

Without a membrane, the bulk fluids' normal stresses would be continuous at the interface, but a membrane can support tension, so the bulk stresses may be discontinuous. We assume that the membrane itself satisfies the Stokes equations and incompressibility, $\hat{\boldsymbol{\nabla}} \cdot \boldsymbol{u}^{m}=0$, where $\hat{\boldsymbol{\nabla}}$ is the gradient operator constrained to the surface $r=R$. This is a well-studied problem for Langmuir monolayers $[29,30]$, but when the membrane is curved there is a new contribution [31] to the force balance relation at the membrane involving its Gaussian curvature $K=R^{-2}$. If $\boldsymbol{e}_{\|}^{ \pm}=e_{r \theta} \hat{\boldsymbol{\theta}}+e_{r \phi} \hat{\boldsymbol{\phi}}$ are the bulk fluids' in-plane normal rates of strain, the boundary condition is

$\eta_{m}\left(\hat{\nabla}^{2} \boldsymbol{u}^{m}+K \boldsymbol{u}^{m}\right)+2\left[\eta_{+} \boldsymbol{e}_{\|}^{+}-\eta_{-} \boldsymbol{e}_{\|}^{-}\right]_{r=R}=\hat{\boldsymbol{\nabla}} \Pi$.

For the case $\eta_{+}=\eta_{-}$, the interior flows that emerge from this calculation match closely those seen in experiment. Cross-sectional profiles shown in Fig. 2(c) at various elevations above the surface agree with the experimental profiles, with significant counterflow both inside and outside the vesicle near its lateral edges, and over much of the lowest cross section. The observed geometry of the internal streamlines [Fig. 2(d)] follows that predicted theoretically [Fig. 2(e)], and the maximum downstream membrane speed is observed at the vesicle apex, as predicted. The fluid motion external to the membrane and the orbiting of domains within the membrane [Figs. 3(a) and 3(b)] are both in agreement with theory. In addition to the circulating motion of the membrane domains we have observed over long periods of time their gradual migration to the two vortex centers on either side of the vesicle midline, leaving a depleted region at the apex [Fig. 3(b)]. This appears to be an example of the motion across streamlines described by Bretherton [32]. Note also the existence of closed streamlines outside the vesicle, as predicted [25].

By plotting the downstream velocity as a function of $z$ through the vesicle apex (Fig. 4), a direct quantitative

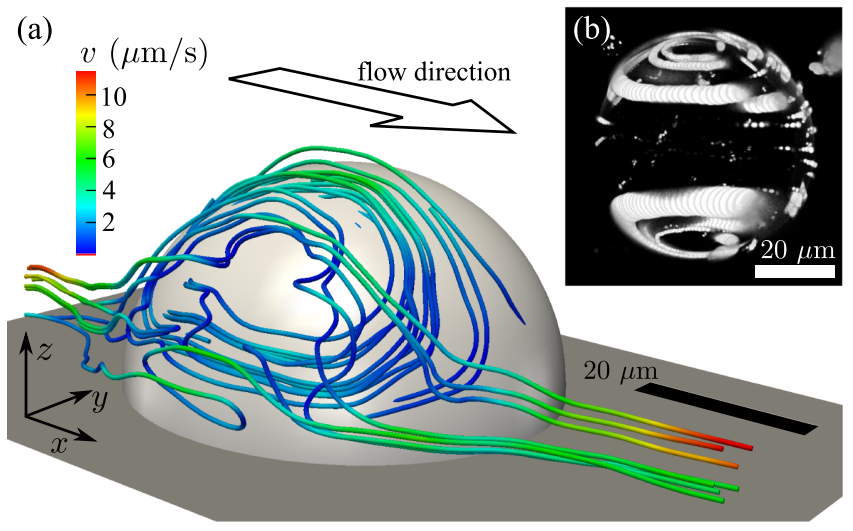

FIG. 3 (color online). Membrane and external flows. (a) Selected external streamlines along one side of an $L_{o}$ vesicle in shear flow, showing closed orbits above the surface. (b) Timelapse confocal stack of an $L_{o}$ vesicle, viewed from above, illustrating circulation of $L_{d}$ domains.

comparison can be made between theory and experiment. The discontinuity in the derivative of the fluid velocity at the membrane, set by the gradient of the membrane tension through Eq. (1), is clearly seen in the downstream velocity as a function of $z$ through the vesicle apex (Fig. 4). This provides perhaps the first direct measurement of tension gradients within bilayer membranes under shear. For the vesicles composed primarily of $L_{o}$ phase the fluid velocity within the vesicle is significantly lower than for $L_{d}$ vesicles as a direct consequence of the greater dissipation in the former, as discussed further below. Returning to the

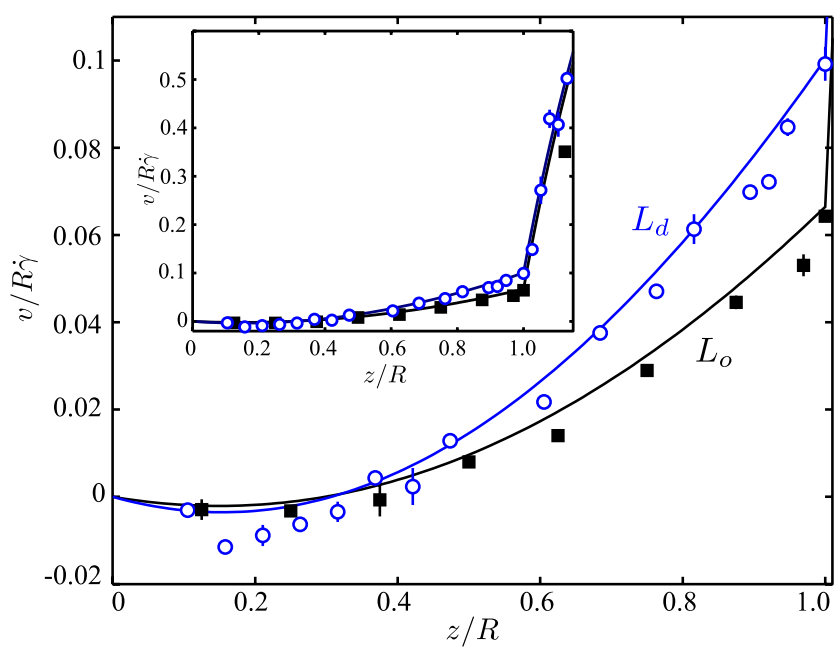

FIG. 4 (color online). Downstream velocity profile through vesicle apex. Data for an $L_{o}$ phase vesicle (black squares) and an $L_{d}$ phase vesicle (blue circles) are scaled by shear rate $\dot{\gamma}$ and vesicle radius $R$, displayed as a function of normalized height above the coverslip surface. PIV experiments (symbols) show quantitative agreement with theoretical velocity predictions (lines) [25]. Inset: extended plot to show the slope discontinuity at the membrane. 
domain tracking in Figs. 1(c) and 1(d), we observe smaller lateral thermal fluctuations in the $L_{o}$ vesicle due to its greater membrane viscosity.

An important empirical result of the calculation [25] is that the speed $v_{0}=\left|\boldsymbol{u}^{m}\right|$ of the membrane at the apex of the vesicle has a simple dependence on $r_{ \pm} \equiv \eta_{m} / R \eta_{ \pm}$, the nondimensional form of the Saffman-Delbrück lengths $\ell_{ \pm} \equiv \eta_{m} / \eta_{ \pm}$[1,31]: $R \dot{\gamma} / v_{0}=A r_{+} / r_{-}+B r_{+}+C$, where $A, B, C$ are known constants. When the inner and outer viscosities are equal (the sucrose and glucose solutions used here had viscosities within $4 \%$ of each other), this result further reduces to the simple linear dependence,

$$
\frac{R \dot{\gamma}}{v_{0}} \simeq 7.86+4.72 \frac{\eta_{m}}{\eta_{w} R}
$$

where $\eta_{w}$ is the viscosity of water. Since $R \dot{\gamma}$ is the fluid velocity in the shear profile in the absence of the vesicle, we term the quantity $R \dot{\gamma} / v_{0}$ the velocity attenuation ratio. Its predicted variation with the Saffman-Delbrück length provides a means of determining $\eta_{m}$ from the apex velocity for a range of vesicle radii.

This analysis shows that in the limit of large vesicle radius or vanishing membrane viscosity, the velocity attenuation ratio is the constant $\sim 7.9$. (A viscosity contrast between internal and external fluids changes the value of the intercept, but the relationship persists in character.) This is a significant and purely geometric effect: the constraint of lateral incompressibility on the membrane velocity field drastically reduces the fluid flow, even in the absence of any internal membrane dissipation. Values of membrane viscosities reported previously $[7,8]$ have ranged from $2-6 \mu$ Poise $\mathrm{cm}$ in disordered lipid phases and from studying dynamic fluctuations near a compositional critical point in membranes $[10,11]$. Values at the lower end would change the velocity attenuation ratio by $\sim 0.5$ for the smallest vesicles that can be studied by this method $(\sim 10 \mu \mathrm{m})$, an increment that is below the noise limit for our measurements. This is seen in the data shown in blue in Fig. 5 for $L_{d}$ membranes. A weighted least squares fit yields an intercept of $8.69 \pm 2.73$, in excellent agreement with the predicted value, and a negligible membrane viscosity $\left(\eta_{m}=1.9 \pm 11 \mu\right.$ Poisecm $)$. This lipid composition thus serves mainly as a control to test the zeroth order velocity attenuation due to membrane incompressibility. On the other hand, the data for the $L_{o}$ phase vesicles show a large change in the attenuation ratio and yield a membrane viscosity of $\eta_{m}=15.7 \pm 9.9 \mu$ Poise $\mathrm{cm}$ (with a consistent intercept of $9.50 \pm 1.41$ ). The overlapping $95 \%$ confidence intervals for extrapolations of both data sets to $R \rightarrow \infty$ indicate clear statistical consistency between the two and with the theoretical prediction.

Finally, we note that recent theoretical work shows that the inclusion of gel domains into a fluid membrane would increase its viscosity to a degree that depends on several factors, including the ratio of domain radius $a$ to the Saffman-Delbrück lengths $\ell_{ \pm}$, and the area fraction of

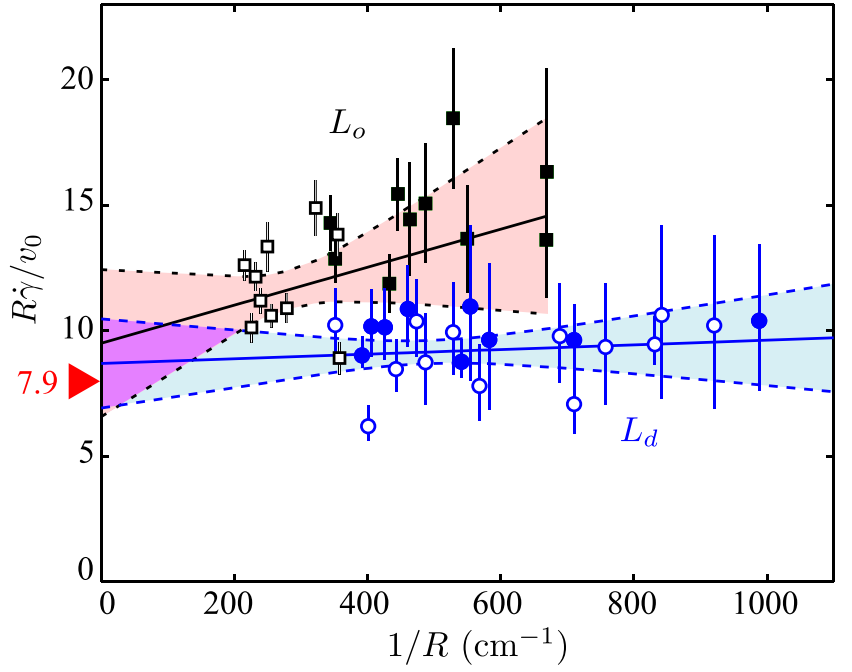

FIG. 5 (color online). Test of the predicted velocity attenuation relation. Scaled membrane velocity $v_{0}$ at vesicle apex for $L_{o}$ phase (black squares) and $L_{d}$ phase (blue circles) as a function of inverse vesicle radius, for $\sim 20$ vesicles in each phase. For each data point, a given vesicle was observed at 2-3 different shear rates to confirm linearity of $v_{0}$ with $\dot{\gamma}$, and the results averaged. Closed symbols indicate vesicles whose height-to-radius ratio was within $10 \%$ of unity. Open symbols deviate by no more than $60 \%$. Black and blue lines indicate weighted least squares fits to the linear relation (2), the slopes of which yield the associated membrane viscosities. Pink and blue shaded areas indicate $95 \%$ confidence intervals, purple indicates overlap. Red arrow indicates predicted intercept.

domains $\phi$ [33]. Domain area fractions for both compositions used here were low, $1-2 \%$ for $L_{o}$ phase vesicles and $1-10 \%$ for $L_{d}$ phase vesicles, and the dilute solution analysis in [33] suggests that our measurements may overestimate the true viscosity by about $4 \%$ in the $L_{o}$ phase and $14 \%$ in the $L_{d}$ phase. Average domain separations were $\sim 2-10 \mu \mathrm{m}$ for both compositions, so our $L_{d}$ membranes did not fully meet the dilute condition required by theory, and hydrodynamic interactions between domains might increase further the apparent viscosity. However, we saw no systematic trend in the measured viscosity of individual vesicles with $\phi$ or domain separation over the small range studied.

In summary, we have presented the first detailed fluid mechanical measurements of the flows inside, on, and around lipid bilayer vesicles under controlled conditions of fluid shear. Detailed analysis of those flow fields for low-viscosity membranes confirms quantitatively a theoretically predicted geometric velocity attenuation effect, and it reveals the scale of membrane viscosity necessary to significantly affect shear-driven flows. The combination of techniques described here may prove useful in the study of more complex systems involving membranes under shear, such as those found in large eukaryotic cells and perhaps in contexts within developmental biology, where the membranes may be more tightly coupled to cytoskeletal 
filaments and the internal cellular rheology may be nonNewtonian.

We thank S. Ganguly, S. L. Keller, and M. Polin for contributions at an early stage of this work and many valuable discussions. This work was supported by the Leverhulme Trust, the Engineering and Physical Sciences Research Council, and the European Research Council Advanced Investigator Grant No. 247333 (R. E. G.).

[1] P. G. Saffman and M. Delbrück, Proc. Natl. Acad. Sci. U.S.A. 72, 3111 (1975); P. G. Saffman, J. Fluid Mech. 73, 593 (1976).

[2] B. D. Hughes, B. A. Pailthorpe, and L. R. White, J. Fluid Mech. 110, 349 (1981).

[3] S. R. Keller and R. Skalak, J. Fluid Mech. 120, 27 (1982).

[4] H. Noguchi and G. Gompper, Phys. Rev. Lett. 93, 258102 (2004); 98, 128103 (2007).

[5] V. Kantsler and V. Steinberg, Phys. Rev. Lett. 95, 258101 (2005); 96, 036001 (2006).

[6] U. Seifert and S. A. Langer, Europhys. Lett. 23, 71 (1993).

[7] R. Dimova, C. Dietrich, A. Hadjiiski, K. Danov, and B. Pouligny, Eur. Phys. J. B 12, 589 (1999); K. D. Danov, R. Dimova, and B. Pouligny, Phys. Fluids 12, 2711 (2000).

[8] E. P. Petrov, R. Petrosyan, and P. Schwille, Soft Matter 8, 7552 (2012).

[9] P. Cicuta, S. L. Keller, and S. L. Veatch, J. Phys. Chem. B 111, 3328 (2007).

[10] B. A. Camley, C. Esposito, T. Baumgart, and F. L. H. Brown, Biophys. J. 99, L44 (2010).

[11] A. R. Honerkamp-Smith, B. B. Machta, and S. L. Keller, Phys. Rev. Lett. 108, 265702 (2012).

[12] G. G. Fuller and J. Vermant, Annu. Rev. Chem. Biomol. Eng. 3, 519 (2012).

[13] R. Shlomovitz, A. A. Evans, T. Boatwright, M. Dennin, and A. J. Levine, Phys. Rev. Lett. 110, 137802 (2013).
[14] M. Sickert, F. Rondelez, and H. A. Stone, Europhys. Lett. 79, 66005 (2007).

[15] A. H. Sadoughi, J. M. Lopez, and A. H. Hirsa, Phys. Fluids 25, 032107 (2013).

[16] J. Verchot-Lubicz and R. E. Goldstein, Protoplasma 240, 99 (2010).

[17] T. Shimmen and E. Yokota, Curr. Opin. Cell Biol. 16, 68 (2004).

[18] R. E. Goldstein, I. Tuval, and J.-W. van de Meent, Proc. Natl. Acad. Sci. U.S.A. 105, 3663 (2008).

[19] J.-W. van de Meent, A. J. Sederman, L. F. Gladden, and R. E. Goldstein, J. Fluid Mech. 642, 5 (2010).

[20] K. Wolff, D. Marenduzzo, and M. E. Cates, J. R. Soc. Interface 9, 1398 (2012).

[21] C. Vézy, G. Massiera, and A. Viallat, Soft Matter 3, 844 (2007).

[22] B. Lorz, R. Simson, J. Nardi, and E. Sackmann, Europhys. Lett. 51, 468 (2000).

[23] E. B. Dussan. V., J. Fluid Mech. 174, 381 (1987).

[24] K. Sugiyama and M. Sbragaglia, J. Eng. Math. 62, 35 (2008).

[25] F. G. Woodhouse and R. E. Goldstein, J. Fluid Mech. 705, 165 (2012).

[26] M. I. Angelova, S. Soleau, P. Meleard, J. F. Faucon, and P. Bothorel, Prog. Colloid Polym. Sci. 89, 127 (1992).

[27] Available at http://www.oceanwave.jp/softwares/mpiv.

[28] C. D. Meinhart, S. T. Wereley, and J. G. Santiago, J. Fluids Eng. 122, 285 (2000).

[29] D. K. Schwartz, C. M. Knobler, and R. Bruinsma, Phys. Rev. Lett. 73, 2841 (1994).

[30] D. K. Lubensky and R. E. Goldstein, Phys. Fluids 8, 843 (1996).

[31] M. L. Henle, R. McGorty, A. B. Schofield, A. D. Dinsmore, and A. J. Levine, Europhys. Lett. 84, 48001 (2008); M. L. Henle and A. J. Levine, Phys. Rev. E 81, 011905 (2010).

[32] F. P. Bretherton, J. Fluid Mech. 14, 284 (1962); See also G. B. Jeffery, Proc. R. Soc. A 102, 161 (1922).

[33] M. L. Henle and A. J. Levine, Phys. Fluids 21, 033106 (2009). 